\title{
The Effects of Cathode Configuration on Hall Thruster Cluster Plume Properties
}

\author{
Brian E. Beal ${ }^{*}$ and Alec D. Gallimore ${ }^{\dagger}$ \\ University of Michigan, College of Engineering, Ann Arbor, MI 48109 USA \\ and \\ William A. Hargus, Jr." \\ Air Force Research Laboratories, Edwards AFB, CA 93524
}

\begin{abstract}
Clusters of Hall thrusters are being considered for use on a variety of missions requiring electric propulsion systems capable of operating at power levels in excess of the current state of the art. One of the key factors to be considered in determining the optimum cluster architecture is the configuration of the electron-emitting cathode(s). This work presents experimentally determined plume properties and discharge current characteristics obtained with multiple thrusters coupled to a single cathode. Spatially resolved plasma density, electron temperature, and plasma potential data are presented during both single thruster and cluster operation. Measurements taken in this configuration are compared to previously published data obtained with each thruster coupled to its own independent cathode. Significant differences between the two configurations are noted and explained. Additionally, critical plasma parameters in the cluster plume are shown to be strongly influenced by the location of the hollow cathode.
\end{abstract}

\section{Nomenclature}

$A=$ Area of one electrode

$A_{S}=$ Surface area of sheath surrounding an electrode

$\mathrm{B}=$ Magnetic field strength

$\mathrm{D}=$ Diffusion coefficient

$\mathrm{e}=$ Electron charge

$\mathrm{E}=$ Electric field strength

$\mathrm{k}_{\mathrm{b}}=$ Boltzmann's constant

$\mathrm{j}_{\mathrm{e}}=$ Electron current density

$\mathrm{m}_{\mathrm{i}}=$ Ion mass

$\mathrm{m}_{\mathrm{e}}=$ Electron mass

$\mathrm{n}=$ Electron number density

$\mathrm{n}_{\mathrm{n}}=$ Neutral number density

$\mathrm{T}_{\mathrm{e}}=$ Electron temperature

$\mathrm{T}_{\mathrm{n}}=$ Neutral temperature

$\mathrm{V}_{\mathrm{d} 2}=$ Voltage measured between triple probe electrodes 1 and 2

$\mathrm{V}_{\mathrm{d} 3}=$ Voltage applied between triple probe electrodes 1 and 3

$\mathrm{V}_{\text {anode }}=$ Anode potential with respect to chamber ground

\footnotetext{
${ }^{*}$ Work performed while student at University of Michigan and employee of ERC, Inc. Currently: Senior Project Engineer, Electric Propulsion Technology, P.O. Box 97009, Redmond, WA 98073, Member AIAA.

${ }^{\dagger}$ Professor, Department of Aerospace Engineering, 1320 Beal Ave., Ann Arbor, MI 48109, Associate Fellow, AIAA

* Research Scientist, Propulsion Directorate, 1 Ara Road, Edwards AFB, CA 93524, Senior Member, AIAA
} 
$\mathrm{V}_{\text {cath }}=$ Cathode potential with respect to chamber ground

$\mathrm{V}_{\text {dis }}=$ Discharge voltage

$\mathrm{V}_{\mathrm{f}}=$ Floating potential

$\mathrm{v}_{\mathrm{D}}=$ Diamagnetic drift speed

$\mathrm{v}_{\mathrm{ExB}}=\mathrm{ExB}$ drift speed

$\delta=$ Sheath thickness

$\lambda_{\mathrm{D}}=$ Electron Debye length

$\sigma_{\text {en }}=$ Electron-neutral collision cross section

$\sigma_{\mathrm{ei}}=$ Electron-ion collision cross section

$\phi=$ Plasma potential

$\mu=$ Electron mobility

$v_{\mathrm{e}}=$ Electron collision frequency

$\omega_{\mathrm{c}}=$ Electron cyclotron frequency

\section{Introduction}

Future space missions will require electric propulsion systems capable of operating at very high power levels compared to those currently in use. ${ }^{1,2}$ One method being considered for reaching these power levels involves clustering multiple devices of moderate power to reach the total throughput desired. An attractive propulsion option for this class of mission is the Hall thruster due to its low specific mass, high thrust density, and high reliability. ${ }^{1,2}$ In an effort to understand the technical issues related to operating multiple Hall thrusters in close proximity to each other, a cluster of four Busek BHT-200-X3 200-watt class devices has been studied in detail and reported on previously. ${ }^{3-7}$

A cluster of thrusters may have a slightly lower efficiency and higher dry mass than a single, similarly powered thruster since larger engines have historically outperformed smaller thrusters. In contrast to this slight potential disadvantage in performance, however, recently published work has concluded that a cluster also offers several advantages over a monolithic thruster. ${ }^{1,2}$ Examples of the benefits of clustering include improved system reliability due to the inherent redundancy of running multiple engines and the ability to throttle the system by simply turning off one or more thrusters. ${ }^{1,2}$ Throttling the system in this way allows the cluster to operate at lower power without running any of the individual thrusters at off-design conditions. This characteristic of a cluster may prove beneficial on missions where either the available power or the propulsive needs change as a function of time. For example, a high-power cluster of Hall thrusters could be used for the initial low-earth orbit to geosynchronous orbit (LEOGEO) transfer of a geosynchronous communications satellite. Upon reaching its final destination, one element of the cluster could then be used for north-south station keeping. In another situation, a cluster of thrusters could be employed on a solar-powered, deep-space probe to provide a high-thrust escape from Earth's gravity well. As the probe progresses away from the sun and the power available from the spacecraft solar arrays decreases, the number of thrusters in operation would decrease accordingly. Through careful design of the appropriate mission architecture, a cluster sized for the initial high-thrust maneuver would ultimately be capable of providing propulsion for a duration well in excess of the lifetime of an individual thruster. A final advantage of clustering is the high degree of system scalability. In principle, once the technical issues involved with operating a cluster are fully understood, a single flight-qualified engine could support a wide range of missions requiring various power levels by simply clustering the appropriate number of thrusters. Thus, enhanced scalability and flexibility make clusters attractive for many missions despite the potential reduction in full-power performance compared to large monolithic thrusters. In light of these advantages, as well as the inherent mass-saving benefits of electric propulsion in general, Aerojet was recently awarded a NASA contract to develop a $600-\mathrm{kW}$ cluster of Hall thrusters in support of the President's Moon/Mars Initiative.

Although using a cluster of high-power thrusters for primary propulsion appears to be advantageous for many missions, including transportation of humans and cargo to the Moon and Mars, there are several systems integration issues that must be considered before clusters can be used in flight. ${ }^{1,2}$ For example, it is imperative that the interaction of the plasma plumes both among the thrusters and with the spacecraft be understood. In an effort to address this issue, the electron number density, electron temperature, and plasma potential downstream of a lowpower cluster were measured using a combination of electrostatic probes. In each case, the profiles recorded in the cluster plume were compared to those measured downstream of an individual thruster. Previous works have demonstrated the methods by which knowledge of these key properties can be used to accurately predict critical 
plasma parameters downstream of a multi-thruster array when each thruster is operated independently, i.e. with its own dedicated hollow cathode and power circuit. ${ }^{6,7}$ In this configuration, analytical methods were shown to be capable of predicting the electron number density, electron temperature, and plasma potential in a cluster plume to within the margin of error of typical plasma diagnostics.

Although the nominal (i.e. independent) cluster configuration considered previously may be preferred in many cases due to its favorable combination of modularity and scalability, there are some situations in which trade studies may show alternative cluster configurations to be advantageous. For example, it may be beneficial in some situations to operate a cluster of thrusters in parallel so that the entire assembly may be powered from a single, large PPU rather than several smaller ones. In other situations, performance benefits may be achieved by operating multiple thrusters from a single cathode. Since propellant injected through the hollow cathode is not accelerated through the engine, it provides no thrust and therefore reduces the overall specific impulse of the system. Clearly, operating multiple thrusters from a single cathode (without increasing the cathode mass flow rate or with an increase that is less than linear with emitted current) would mitigate the effects of this loss mechanism compared to operating each thruster with its own cathode. Although reliability considerations almost certainly eliminate the possibility of using a single cathode for neutralization of an entire multi-thruster array in an operational cluster design, a reconfigurable system that could support shared cathode operation in the event of a single-unit failure would provide significant risk reduction for spacecraft designers. This article examines some of the technical issues and challenges related to each of these alternative configurations.

\section{Experimental Apparatus}

\section{A. Cluster}

The cluster used in this experiment was composed of four Busek BHT-200-X3 200-watt class Hall thrusters. An earlier version of this thruster was reported to operate at an anode efficiency of $42 \%$ and specific impulse of 1300 seconds while providing $12.4 \mathrm{mN}$ of thrust at the nominal operating conditions. ${ }^{8}$ Each thruster had a mean discharge channel diameter of $21 \mathrm{~mm}$ and was operated on xenon propellant. The thrusters were arranged in a $2 \times 2$ grid with approximately 11.4 centimeters between the centerlines of nearest neighbors. Typical operating conditions for the BHT-200 are given in Table 1. Figure 1 shows the cluster during operation.

\begin{tabular}{|c|c|}
\hline Parameter & Value \\
\hline Discharge Voltage (V) & $250 \pm 0.5$ \\
\hline Discharge Current (A) & $0.80 \pm 0.03$ \\
\hline Cathode Voltage (V) & $-8.5 \pm 1.0$ \\
\hline Magnet Current (A) & $1.0 \pm 0.03$ \\
\hline Keeper Current (A) & $0.5 \pm 0.05$ \\
\hline Keeper Voltage (V) & $13 \pm 1$ \\
\hline Anode Mass Flow (sccm) & $8.5 \pm 0.85$ \\
\hline Cathode Mass Flow (sccm) & $1.0 \pm 0.1$ \\
\hline
\end{tabular}

Table 1. Typical operating parameters of the BHT-200.



Figure 1. Two views of a low-power Hall thruster cluster during operation. 
The naming convention and coordinate system used throughout this experiment are shown in Fig. 2. As shown, the thrusters were labeled as TH 1-4 beginning in the upper left-hand corner and proceeding counterclockwise when viewed from downstream. The origin of the coordinate system was defined as the midpoint of the cluster in the displayed $\mathrm{X}-\mathrm{Y}$ plane. The $\mathrm{Z}$ coordinate measured the distance downstream of the thruster exit plane. A threedimensional positioning system was used to sweep probes through the plasma plume.

Several different experimental configurations were tested to explore the various modes of cluster operation discussed in the previous section. In the first arrangement, both thrusters 2 and 3 were operated from a single discharge power supply, as sketched in Fig. 3. The main goal of operating the thrusters in parallel was to examine the possibility of cathode current sharing between the devices through the plasma plume. The electromagnet, keeper, and cathode heater circuits remained separate between the thrusters. The current emitted by each cathode was measured using powered Hall effect sensors.

In the second experimental configuration, two thrusters were operated from a single hollow cathode to examine the effects of cathode number and placement on plume properties. This was accomplished with two separate cathode arrangements. In one case, two thrusters were operated from cathode 3. Measurements were conducted at the Air Force Research Laboratory (AFRL) with thrusters 3 and 4 operating from cathode 3 , while the shared cathode tests at the Plasmadynamics and Electric Propulsion Laboratory (PEPL) used thrusters 2 and 3 simply because of the different probe positioning systems used in these facilities. In both facilities, the xenon flow rate through the cathode remained constant at $1 \mathrm{sccm}$. The second neutralizer tested in this "shared cathode" configuration was a 6.35 -

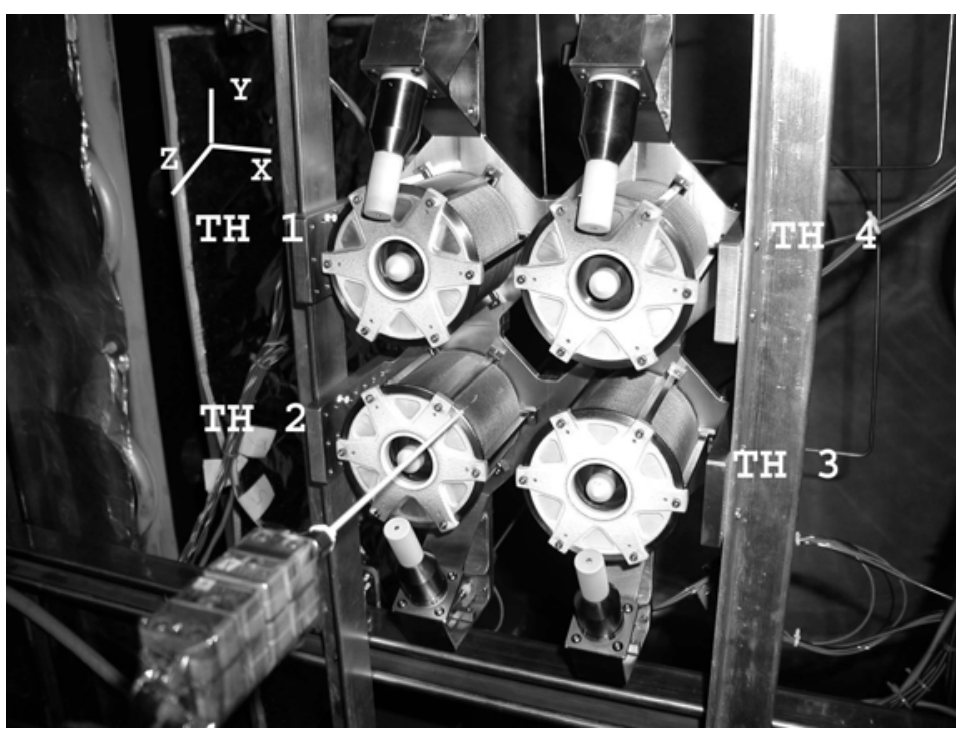

Figure 2. The thruster naming convention and coordinate system used throughout this paper. For some of the tests reported here, an additional cathode (not shown) was located at the geometric center of the cluster. mm (1/4") Model HCN-252 hollow cathode available from Ion Tech, Inc. It was placed at the center of the cluster and operated with a constant $5 \mathrm{sccm}$ xenon flow rate. Since there is no reason to suspect that the different cathode designs have any significant effect on the operation of the engines, comparing data obtained with the Ion Tech cathode to measurements made using the shared Busek cathode allows the effect of cathode location to be examined. The discharge circuit used during the shared cathode measurements is sketched in Fig. 4.

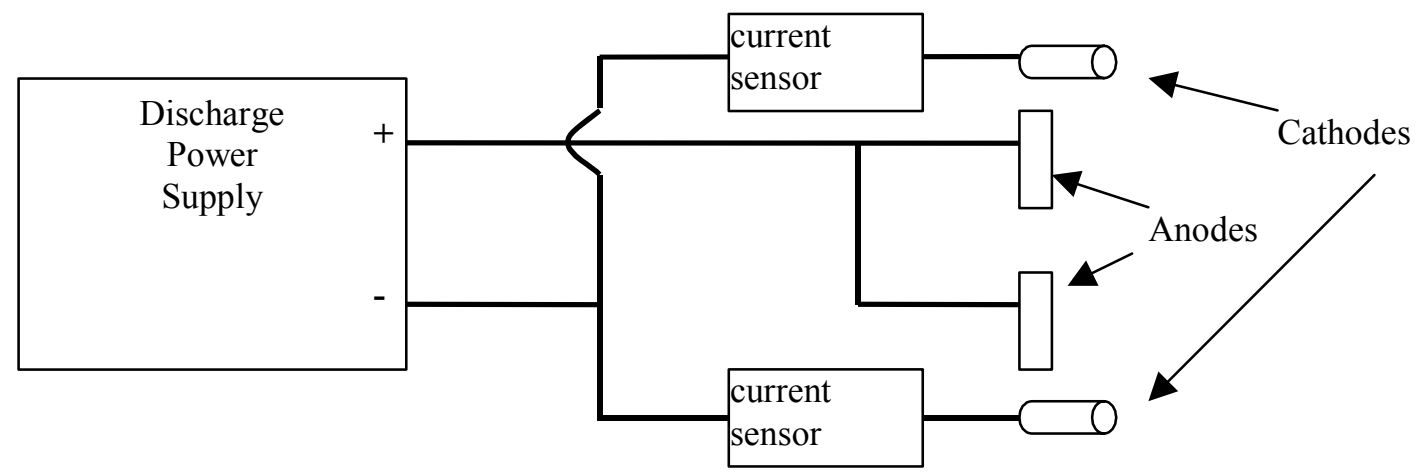

Figure 3. A simplified sketch of the discharge circuit used to study parallel operation of multiple thrusters.

4

American Institute of Aeronautics and Astronautics

Distribution A: Approved for public release / distribution unlimited. 


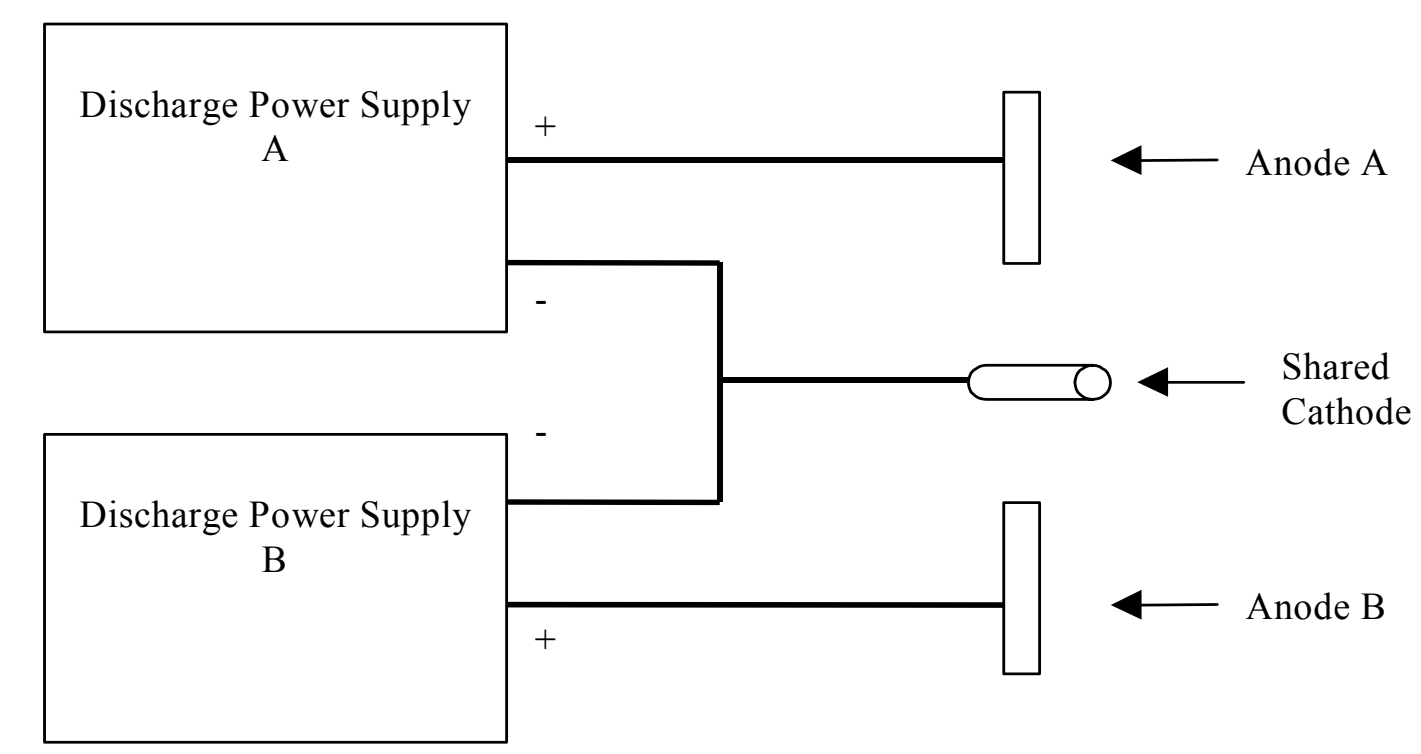

Figure 4. A simplified sketch of the discharge circuit used to examine shared cathode operation.

\section{B. Vacuum Facilities}

Two different vacuum facilities were used for various portions of the tests described here. The first was the Large Vacuum Test Facility (LVTF) at the University of Michigan. The LVTF is a stainless steel-clad, cryopumped chamber that is 6 meters in diameter, 9 meters long, and is described in detail elsewhere. ${ }^{6}$ The LVTF features a maximum pumping speed of 240,000 liters per second on xenon and achieves a typical base pressure of approximately $2.5 \times 10^{-7}$ Torr. For the tests reported here, only four of the seven available cryopumps were used resulting in chamber background pressures ranging from $1.1 \times 10^{-6}$ for single-thruster operation to $3.6 \times 10^{-6}$ Torr (corrected for xenon) during operation of all four thrusters.

The second vacuum facility used in these experiments was Chamber 6 at AFRL. Chamber 6 is a $1.8 \times 3.0$ meter cylindrical, stainless steel vacuum chamber that is evacuated by one dual-stage cryopump and four single-stage cryopanels. During thruster operation, the chamber pressure stabilized at approximately $6.1 \times 10^{-6}$ Torr for single thruster operation and $2.3 \times 10^{-5}$ Torr for four-thruster operation. Both reported pressures are corrected for xenon.

\section{Triple Probe}

The symmetric triple probe, originally developed by Chen and Sekiguchi, ${ }^{9}$ is a convenient plasma diagnostic for collecting large amounts of electron temperature and density data due to the elimination of the voltage sweep required by other electrostatic probes. Additionally, since the probe as a whole floats, the disturbance to the ambient plasma is minimized compared to single Langmuir probes, which draw a net current from the discharge. The triple probe used for this experiment consisted of three tungsten electrodes insulated from each other by an alumina rod. The diameter of each electrode was $0.50 \mathrm{~mm}$ and the length extending past the end of the alumina was $5.0 \mathrm{~mm}$. The electrodes were aligned parallel to the axis of each thruster and spaced approximately two electrode diameters apart. The probes were sized to criteria that allowed the standard thin sheath assumptions of

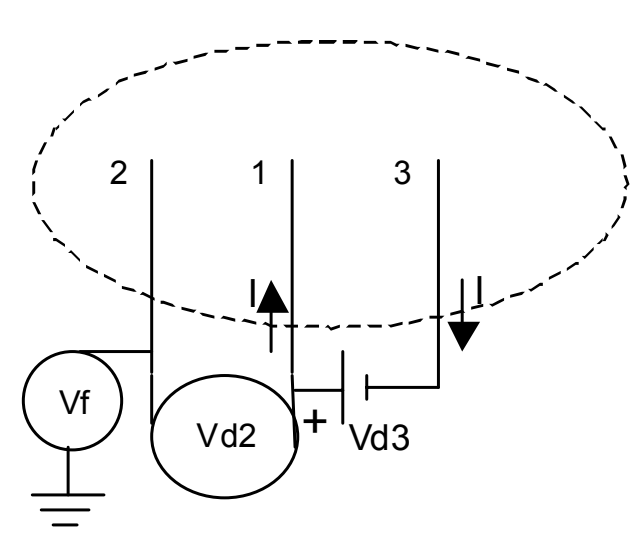

Figure 5. The triple probe circuit used for measurement of plasma density and electron temperature. 
probe theory to be applied. ${ }^{10}$ Further, it was assumed that the electrodes were sufficiently separated to avoid interaction with each other and that the spatial gradients of plasma properties were sufficiently small to ensure that all three electrodes were exposed to identical plasmas. A schematic of the triple probe circuit is shown in Fig. 5. In this work, electrode 2 was allowed to float while the voltage, $V_{\mathrm{d} 3}$, was applied by a laboratory power supply with floating outputs. For the tests reported here, $\mathrm{V}_{\mathrm{d} 3}$ was set to 12 volts. The probes were numbered in order of decreasing potential such that probe 2 was at the floating potential while probes 1 and 3 were biased above and below the floating potential, respectively.

The method used to determine plasma properties from measured probe data begins with the probe characteristics derived by Chen and Sekiguchi and given in Eqns. 1-2. ${ }^{9}$ In these equations, $\mathrm{n}_{\mathrm{e}}$ is the electron number density, which is equal to the ion number density through the quasineutrality assumption. The electron temperature is represented by $T_{e}$, and ion and electron masses are denoted by $m_{i}$ and $m_{e}$, respectively. The symbol A denotes the surface area of a single electrode, e is the electron charge, and $\mathrm{k}_{\mathrm{b}}$ is Boltzmann's constant. Equations 1 and 2 are first used to obtain an estimate of the electron number density and electron temperature. These estimates are then used to determine a value of the sheath thickness, $\delta$, surrounding each electrode based on the derivation given by Hutchinson and shown in Eqn. 3, where $\lambda_{\mathrm{D}}$ is the electron Debye length. ${ }^{11}$ The sheath thickness, according to this method, is approximately 5 Debye lengths for a xenon plasma. The value of thickness given by Eqn. 3 is then used to calculate the surface area of the sheath around each electrode, $A_{S}$, according to Eqn. 4, where $r_{p}$ is the probe radius. This updated value of the effective electrode collection area is substituted back into Eqn. 1 to determine an updated value of the plasma density, and the routine proceeds iteratively until the solution converges.

$$
\begin{gathered}
n=\left(\frac{e m_{i}}{k_{b} T_{e}}\right)^{1 / 2} \frac{I \exp \left(\frac{1}{2}\right)}{A e^{3 / 2}\left[\exp \left(\frac{e V_{d 2}}{k_{b} T_{e}}\right)-1\right]} \\
\frac{1-\exp \left(\frac{-e V_{d 2}}{k_{b} T_{e}}\right)}{1-\exp \left(\frac{-e V_{d 3}}{k_{b} T_{e}}\right)}=\frac{1}{2} \\
\delta=1.02 \lambda_{D}\left[\left(\frac{1}{2} \ln \left(\frac{m_{i}}{m_{e}}\right)\right)^{1 / 2}-\frac{1}{\sqrt{2}}\right]^{1 / 2}\left[\left(\frac{1}{2} \ln \left(\frac{m_{i}}{m_{e}}\right)\right)^{1 / 2}+\sqrt{2}\right] \\
A_{S}=A\left(1+\frac{\delta}{r_{p}}\right)
\end{gathered}
$$

The method of data analysis used in this article is believed to provide measurements that are of essentially constant validity over most of the sampled region by explicitly accounting for the slight variations in the effective collection area of the probe that occur due to changes in the sheath thickness. Various previously published error analyses indicate that the absolute uncertainty in the calculated electron temperature and number density for typical triple probes are generally less than $30 \%$ and $60 \%$, respectively. ${ }^{9,12}$ The relative uncertainty between two data points recorded using the same probe is believed to be significantly lower than the absolute uncertainty because many 
potential sources of error (i.e., uncertainty in probe dimensions, slight asymmetry of the electrodes, etc.) remain constant over the entire spatial region.

\section{Emissive Probe}

Plasma potential measurements were conducted using a floating emissive probe similar to the one described by Haas, et al. ${ }^{13}$ The emitting portion of the probe consisted of a loop of $0.13 \mathrm{~mm}$ diameter tungsten filament, the ends of which were inserted into double bore alumina tubing along with $0.51 \mathrm{~mm}$ diameter molybdenum wire leads. Short lengths of tungsten wire were inserted into the alumina tube to insure contact between the emitting filament and molybdenum leads. The diameter of the emitting filament loop was approximately $3 \mathrm{~mm}$. Fig. 6 shows a sketch of the emissive probe and control circuit. The normal to the plane of the loop formed by the emitting filament was oriented in the $\mathrm{X}$ direction shown in Fig. 2.

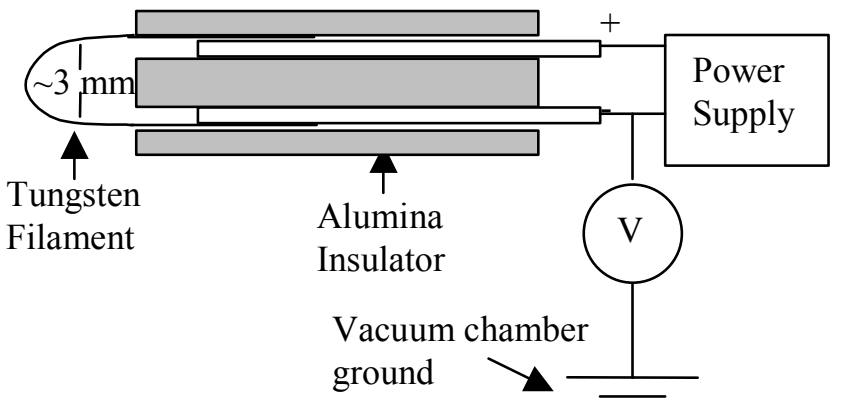

Figure 6. The emissive probe and circuit used for plasma potential measurements.

The theory of the emissive probe is well established and results in the conclusion that a thermionically emitting filament in a low-temperature plasma will approach the local plasma potential when its emitted electron current is sufficient to neutralize the plasma sheath. ${ }^{14}$ For this experiment, the current necessary to heat the probe was provided by a programmable power supply with floating outputs. At each location in the plume, the current was steadily increased and the potential with respect to ground at the negative terminal of the power supply was recorded. This method allowed for verification of a well-defined plateau in the voltage-current trace indicating neutralization of the plasma sheath. Considering that the voltage drop across the emitting filament never exceeded 6 $\mathrm{V}$ and the potential was measured at the negative terminal of the probe, the absolute uncertainty in the plasma potential measurements is estimated to be $-3,+6 \mathrm{~V}$. The relative uncertainty between data points obtained using the same probe is believed to be significantly smaller than this value because the main source of uncertainty, the $\sim 5 \mathrm{~V}$ potential difference across the emitting filament, remained nearly constant over the entire sampled range. The relative uncertainty between data points is therefore conservatively estimated to be $\pm 1.5 \mathrm{~V}$ and is dominated by variations in electron temperature that can influence the small potential drop across the sheath surrounding the emitting filament.

\section{Results}

\section{E. Discharge Current Characteristics}

Discharge current characteristics recorded with two thrusters (TH2 and TH3) operating in parallel are shown as a function of time in Fig. 7. As shown, the current flowing through each anode is approximately 0.80 amps and is nearly constant between the thrusters. This is to be expected since the anode current is controlled primarily by the propellant mass flow rate through each engine. The cathode current traces, on the other hand, show distinct differences between the two units with cathode 3 supplying nearly all of the current necessary to operate both engines. The source of this dominance is not entirely clear, but may be due to minor variations between the cathodes resulting in one having a slightly lower affinity for electron emission. This creates a higher effective resistance for current flowing through that cathode and electrons, choosing the path of least resistance, flow preferentially through cathode 3 . In Fig. 7, the constant $0.50 \mathrm{amp}$ keeper current flowing through each cathode has been subtracted from the displayed traces. The high current levels recorded during the first few minutes of operation were due to operation of the cathode heaters, which were turned off after the system reached steady-state operation. 


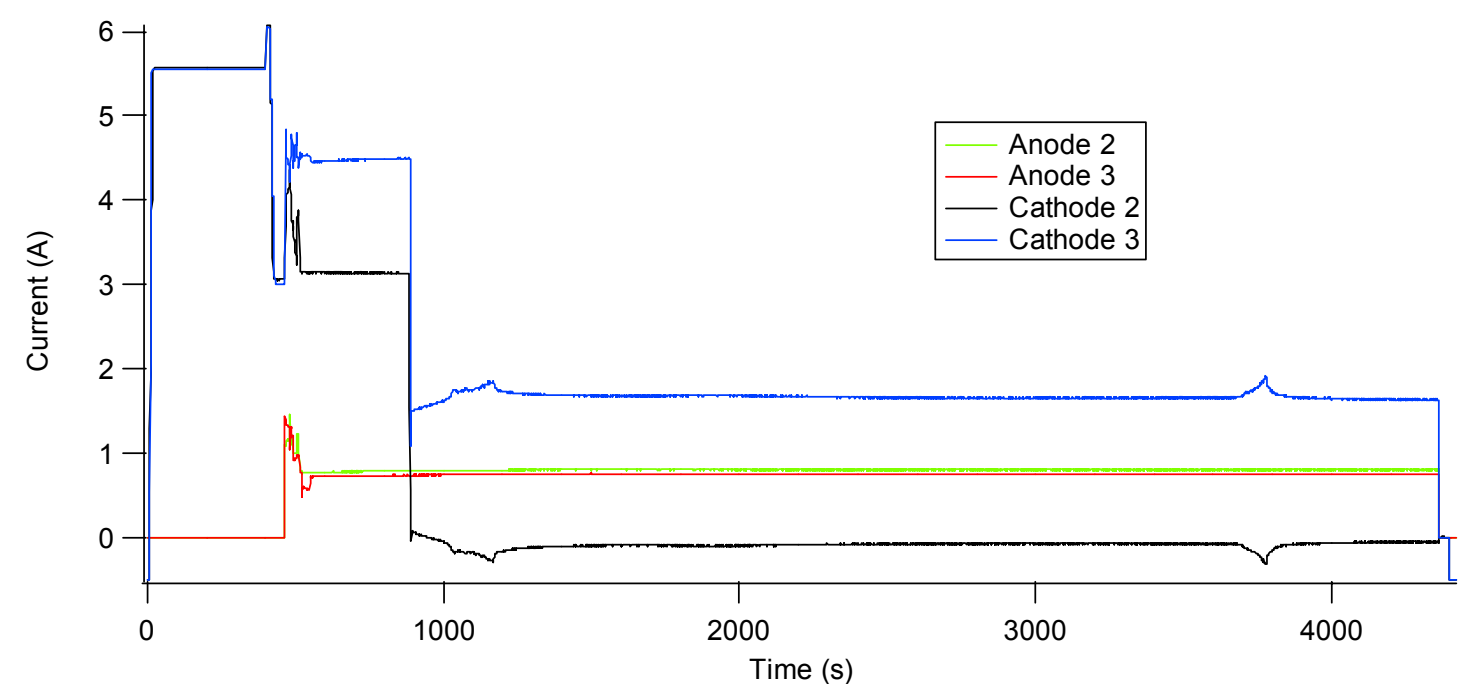

Figure 7. Anode and cathode currents measured during parallel operation of TH2 and TH3 from a single power supply. Note the large fraction of the total current emitted by cathode 3 despite the fact that both cathodes were operated in an identical manner (i.e. with identical mass flow rates and in equivalent locations relative to the thrusters).

The dominance of one cathode displayed in Fig. 7 has potentially important implications for cluster design. In particular, it implies that each cathode in a cluster of thrusters intended for parallel operation should be capable of supplying sufficient current to neutralize the entire cluster since it is doubtful that variability in the key parameters of various cathodes could be reduced enough to prevent one cathode from dominating the discharge. Drawing sufficient charge from a single cathode is not particularly challenging for a low-power cluster with a total current throughput of only a few amps, but for the very high-power systems in which clusters will likely be implemented (e.g. the $600-\mathrm{kW}$ array currently under development) emission of the entire cluster current from a single hollow cathode may prove to be impractical. In this case, the power processing unit (PPU) may need to be modified to ensure that the current flowing through each cathode remains at an acceptable level. The added complexity associated with the current balancing circuitry or active control system could potentially negate any performance and mass advantages associated with using a single, large PPU instead of several smaller ones. The result observed here has implications for the design of large monolithic thrusters, as well as clusters. Based on the data shown in Fig. 7, it can be concluded that operation of a single thruster with multiple smaller cathodes intended to jointly emit the required electron current is likely to be unsuccessful unless precautions are taken to ensure equal loading.

Turning our attention to the case where two thrusters were operated with individual power supplies and a single, shared cathode, it was found that no particularly interesting or

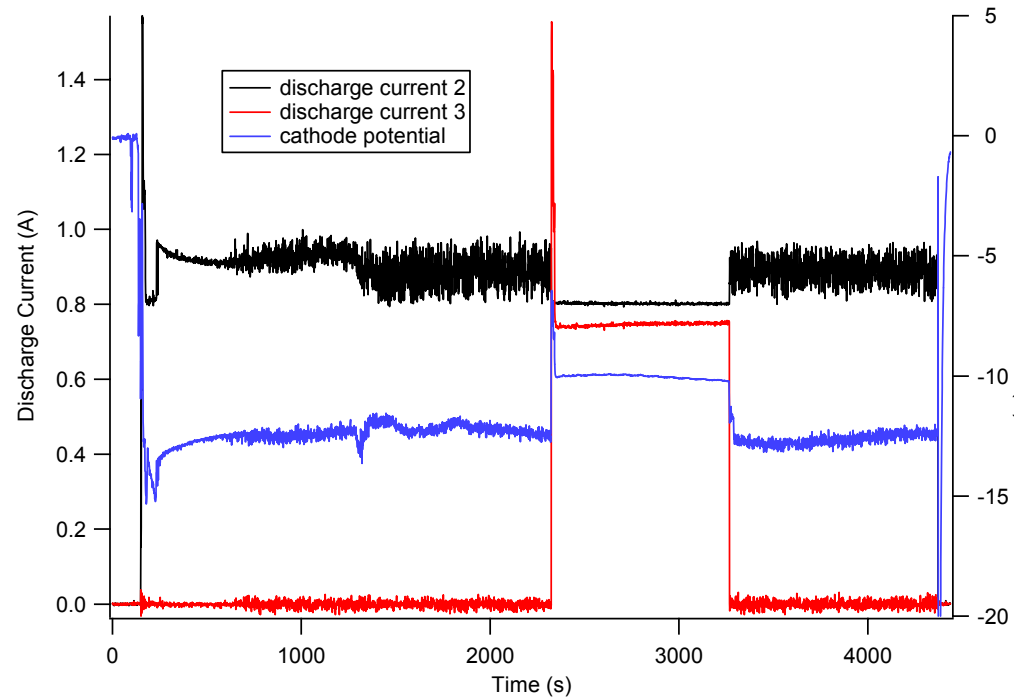

Figure 8. Discharge current characteristics with two thrusters coupled to a single cathode. Note the distinct difference in TH2 current oscillations depending on whether or not $\mathrm{TH} 3$ was in operation. 
surprising phenomena occurred when both thrusters were operated simultaneously. Running a single thruster from a distant cathode, on the other hand, caused significant changes in operating conditions. The discharge current and cathode potential data displayed in Fig. 8 were obtained with both TH2 and TH3 coupled to cathode 3 in the LVTF. As shown, when TH 2 was operated alone with cathode 3 (i.e. from Time $=0$ to approximately Time $=2300$ seconds), the discharge current was slightly higher than normal and the magnitude of current oscillations was also higher than observed in the nominal configuration. ${ }^{6}$ This is consistent with previously published measurements that showed the electromagnetic noise radiated from a larger Hall thruster to increase as the distance between the engine and cathode was increased. ${ }^{15}$ When TH3 was ignited (at approximately Time $=2300$ seconds), the discharge current and magnitude of oscillations in TH2 decreased to near nominal levels. At the same time, the cathode potential increased (moved closer to ground) by about 2.5 volts, thus bringing it to near the nominal level. When TH3 was then shut off (at approximately Time $=3200$ seconds) without changing any settings to $\mathrm{TH} 2$, the discharge current and cathode potential returned to their original, anomalous values. Possible causes of this behavior will be discussed in Section 4.

\section{F. Plasma Density}

The triple probe was used to measure the plasma density in the plume for both shared cathode configurations: with the Ion Tech cathode shared and with cathode 3 shared. Measurements were obtained in Chamber 6 with thrusters 3 and 4 operating individually and simultaneously. Figures 9 through 11 show the profiles recorded at five different axial locations in the plume. Although these plots each contain a large amount of data, the colors and symbols have been chosen to enhance clarity. As the legend shows, all of the blue traces were obtained with the thrusters sharing the Ion Tech cathode located at the center of the cluster. The data recorded with cathode 3 shared are depicted in red. Measurements made with thruster 3 operating alone are represented by diamonds, thruster 4 by circles, and both thrusters operating together by triangles. The thick black line in each figure depicts the density profile measured with each thruster operating in conjunction with its own Busek cathode, i.e. in the nominal configuration reported previously. ${ }^{6,7}$

The plasma density measurements shown below reveal several interesting features related to shared cathode operation. First, the density downstream of a cluster operating with a single neutralizer cannot be predicted by simply summing the contributions from each individual thruster, as they can in the completely modular configuration. ${ }^{6,7}$ This is particularly evident from examination of the data taken with cathode 3 shared. In this situation, thruster 3 shows no unusual plume characteristics when operating alone, which is to be expected since it is coupled to its own cathode. When thruster 4 is operated from this same cathode, however, the plume appears very diffuse and the peak density is more than a factor of 10 lower than the one measured with the engine coupled to its own cathode. Most surprising is that the density downstream of thruster 4 increases to near the nominal profile (within about 25\%) when TH $3 \& 4$ are operated simultaneously. Clearly, operating both thrusters together changes the basic operation of thruster 4 , thus eliminating the possibility of predicting the cluster plume via superposition. Incidentally, the data presented here confirm the previous statement that it is the location of the hollow cathode and not the specific design of the electron emitter that causes changes in the plume properties. This is obvious since the profile downstream of thruster 4 differs greatly from that of thruster 3 when each is operated individually with cathode 3. Increasing the distance between the thruster and the neutralizer seems to dramatically decrease the plasma density in the plume.

Examination of the data taken with the thrusters coupled to the central Ion Tech cathode reveals similar trends to those discussed above. Since this cathode is significantly farther away from the anode of each thruster than the cathodes of the nominal configuration, the lower density observed in the plume with each thruster running individually is consistent with the observations reported above. When both thrusters are operated together, the peak density downstream of each engine increases significantly compared to the level measured during individual

operation. The plasma density with both thrusters operating from the central cathode, however, falls short of the ones measured with cathode 3 shared as well as those measured in the nominal configuration. 


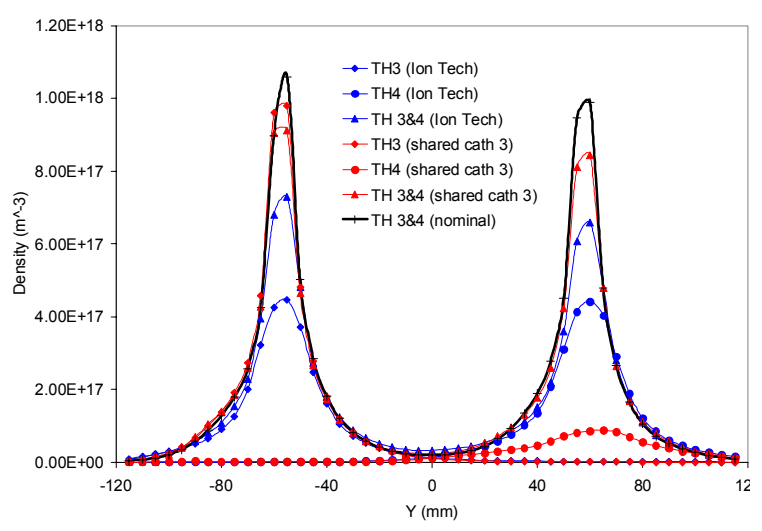

Figure 9. Plasma density recorded $50 \mathrm{~mm}$ downstream of $\mathrm{TH} 3$ and $\mathrm{TH} 4$ for various cathode coupling conditions. Note the low plasma density downstream of a thruster operated from a distant cathode.



Figure 11. Plasma density measured $150 \mathrm{~mm}$ downstream of TH3 and TH4 for various cathode configurations.

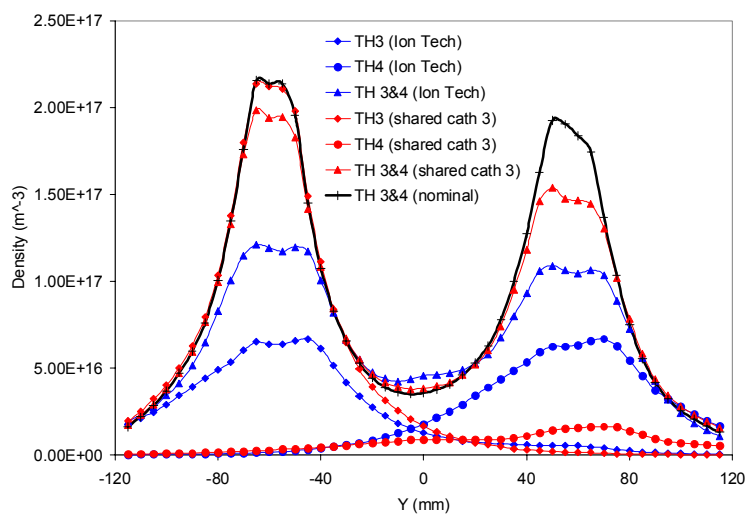

Figure 10. Plasma density profiles measured 100 mm downstream of $\mathrm{TH} 3$ and $\mathrm{TH} 4$ for various cathode configurations. Red traces were recorded with both thrusters coupled to cathode 3 ; blue traces were recorded during operation of the cathode in the center of the cluster.

While Figs. 9-11 show clearly that the location of the cathode has a significant effect on the properties in the plasma plume, they do not explain why this is the case. To provide a more extensive database for studying possible causes, several additional sets of measurements were obtained at PEPL with thrusters 2 and 3 coupled to cathode 3 . The configurations tested were:

(1) TH2 running alone.

(2) $\mathrm{TH} 2$ running and propellant flowing through $\mathrm{TH} 3$ (without a discharge). Testing with propellant flowing through thruster 3 allows the effect of collisions to be evaluated (qualitatively, at least) by increasing the local neutral density in the region between cathode 3 and thruster 2 .

(3) $\mathrm{TH} 2$ running with propellant flowing through $\mathrm{TH} 3$ and electromagnet 3 energized.

(4) Thrusters 2 and 3 operating simultaneously from cathode 3 .

The plasma density profiles recorded at three different locations downstream of thrusters 2 and 3 at PEPL are displayed in Figs. 12-14 below. As shown in these plots, operating thruster 2 alone with cathode 3 resulted in a very diffuse plume with a low plasma density in agreement with the behavior discussed above. The addition of flow through thruster 3 , and the concomitant increase in local pressure, caused the density in the plume to increase by about a factor of two, although it remained far below the levels exhibited during normal operation. Energizing the electromagnet of thruster 3 had no discernible effect. Finally, igniting thruster 3 caused the plasma density downstream of both thrusters to increase dramatically to levels consistent with those reported previously for operation in the independent, modular configuration. ${ }^{6,7}$ 


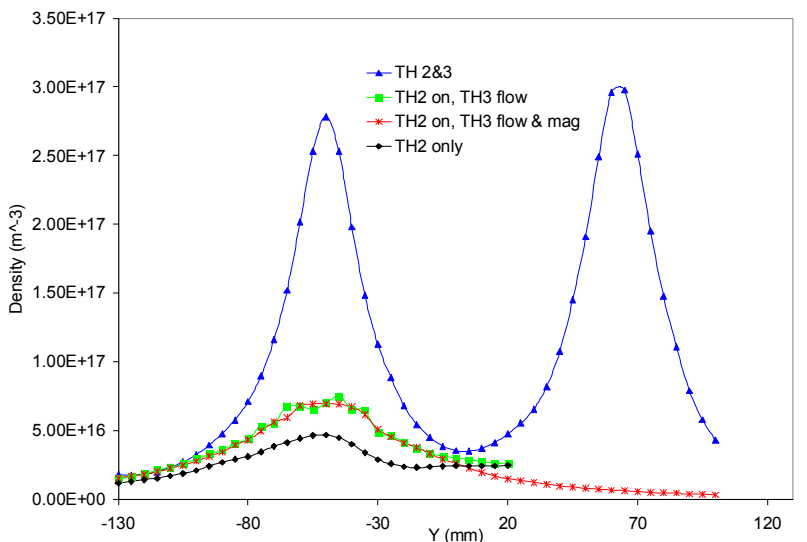

Figure 12. Plasma density profiles measured $70 \mathrm{~mm}$ downstream of TH2 and TH3 for various operating parameters. Both thrusters were coupled to cathode 3 .

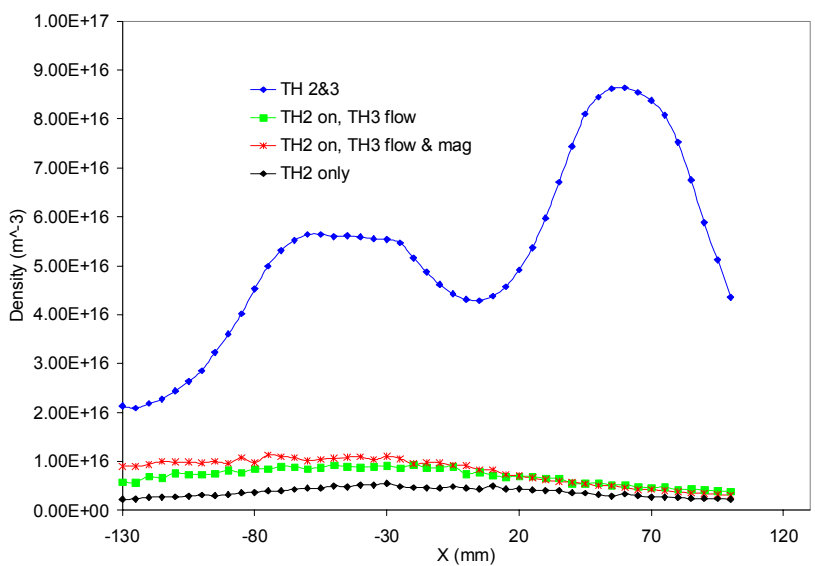

Figure 14. Plasma density profiles $120 \mathrm{~mm}$ downstream of two thrusters operating from a shared cathode.

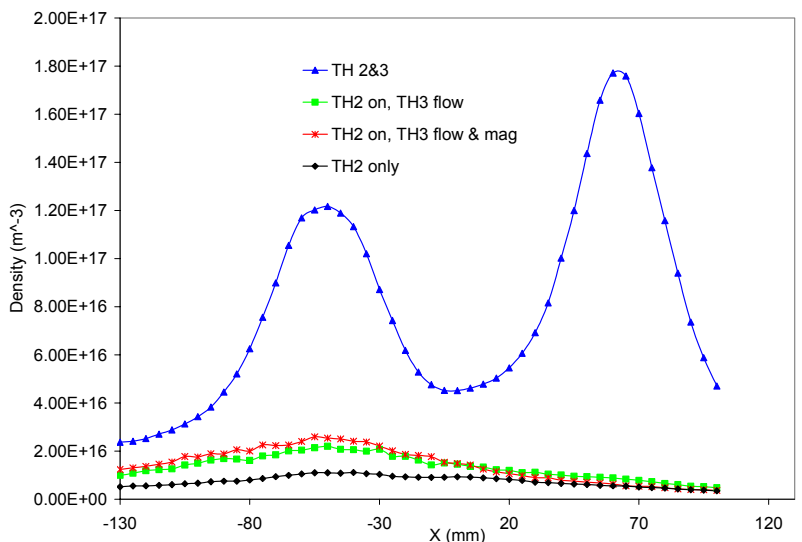

Figure 13. Plasma density profiles measured $120 \mathrm{~mm}$ downstream of TH2 and TH3 during operation from a shared cathode.

\section{G. Electron Temperature}

The same triple probe used to obtain the density measurements presented in the previous section also gave the local electron temperature. Figures 15 through 17 show the electron temperatures measured in Chamber 6 at AFRL for the two different shared cathode experiments. As shown, the electron temperature downstream of a thruster tended to increase when it was operated with a distant cathode. For example, when TH4 was operated in conjunction with cathode 3, Fig. 15 shows that the temperature peaked at over $10 \mathrm{eV}$ compared to approximately $3 \mathrm{eV}$ during operation with a normally-positioned cathode. ${ }^{6,7}$ Coupling to the Ion Tech cathode in the center of the cluster caused similar behavior and the peak electron temperature with one engine running rose to approximately $6 \mathrm{eV}$. As expected, the peak electron temperature decreased with increasing downstream distance. Even at an axial distance of $150 \mathrm{~mm}$, or approximately 7 thruster diameters, the temperature downstream of TH4 remained approximately a factor of two higher when operated from a distant cathode compared to a local one. Regardless of which cathode was used, running multiple thrusters tended to reduce the electron temperature in the plume, bringing it closer to the normal level. Operating both thrusters in conjunction with cathode 3 caused the electron temperature to fall to almost exactly the nominal values, while it remained somewhat above normal during operation of the Ion Tech cathode. $^{6}$

Electron temperatures measured at three axial locations in the LVTF with thrusters 2 and 3 sharing a single Busek cathode are shown in Figs. 18-21. As expected from the measurements obtained in Chamber 6, operating TH2 with the distant cathode 3 caused the electron temperature in the plume to rise well above the values measured in the nominal configuration. ${ }^{6}$ In this mode, the temperature along the centerline of TH2 was approximately $6.5 \mathrm{eV}$ at $\mathrm{Z}=70 \mathrm{~mm}$ and fell to less than $2.5 \mathrm{eV}$ by $170 \mathrm{~mm}$ downstream of the exit plane. When an $8.5 \mathrm{sccm}$ propellant flow was initiated through thruster 3 (without igniting a discharge), the electron temperature downstream of TH2 fell to about $3.5 \mathrm{eV}$ at $70 \mathrm{~mm}$ and $1.5 \mathrm{eV}$ by $170 \mathrm{~mm}$ downstream. This is similar to the behavior of the plasma density, which also showed significant changes when the average neutral density between the thruster and cathode was increased. Energizing the electromagnet of thruster 3 had very little effect on the temperature in the plume. When 
thruster 3 was operated in conjunction with thruster 2, the electron temperature fell to nominal levels and exhibited a high degree of symmetry between the plumes of the two engines, despite the fact that the hollow cathode was much closer to TH3 than it was to TH2. It can therefore be said that increasing the local pressure and running multiple thrusters both tend to decrease the electron temperature in the plume for clusters operated with a single cathode. A possible explanation for this behavior will be discussed in Section IV.

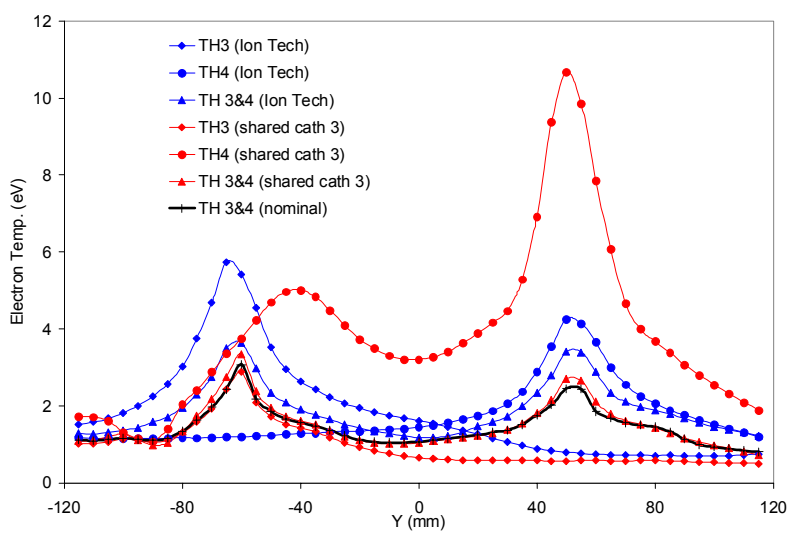

Figure 15. Electron temperature profiles measured $50 \mathrm{~mm}$ downstream of TH3 and TH4 in Chamber 6. Note the high electron temperature downstream of TH4 (at Y=57.5 mm) when operated from cathode 3 .

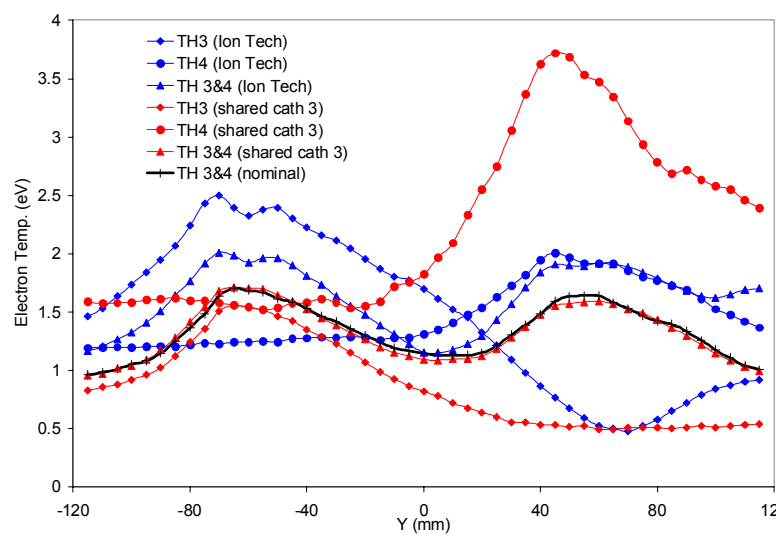

Figure 16. Electron temperature profiles recorded $100 \mathrm{~mm}$ downstream of TH3 and TH4 for various cathode configurations.

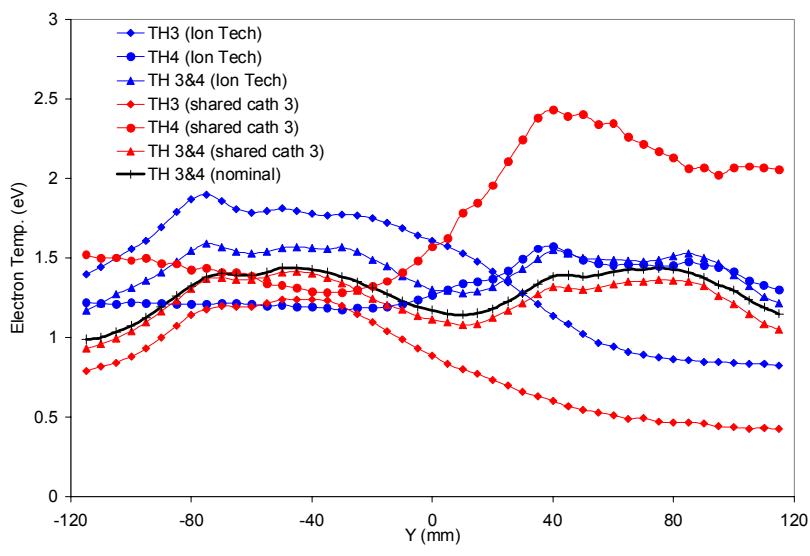

Figure 17. Electron temperature profiles $150 \mathrm{~mm}$ downstream of TH3 and TH4.

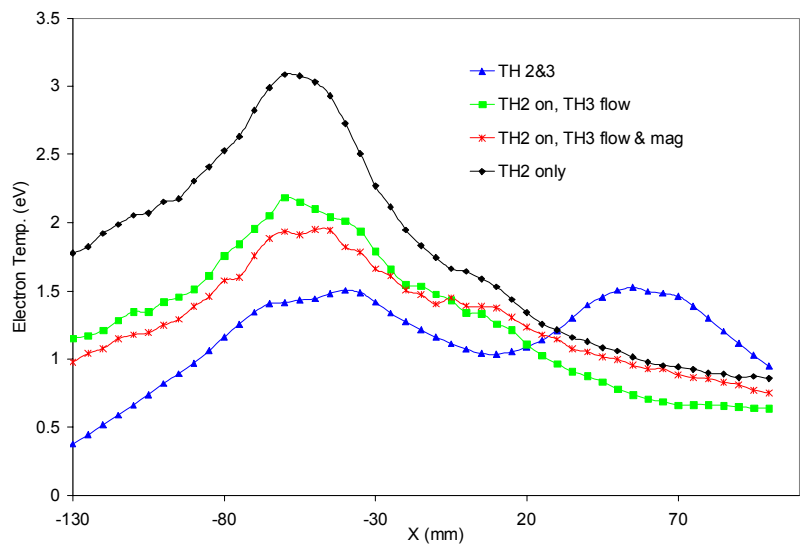

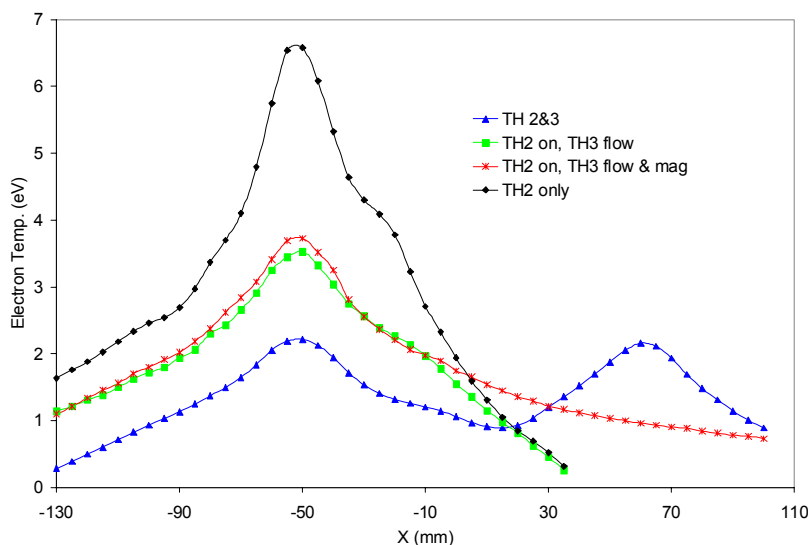

Figure 18. Electron temperature measured at $Z=70$ mm with TH2 and TH3 coupled to cathode 3.

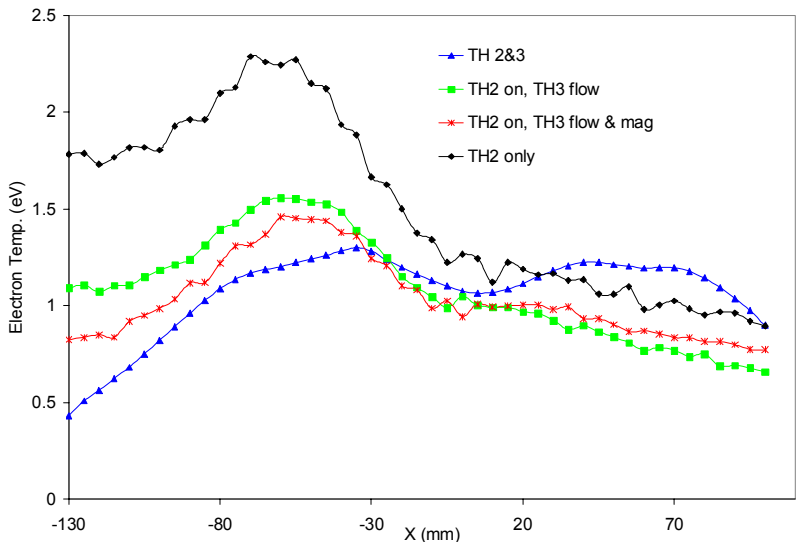

Figure 19. Electron temperature profiles at $Z=120 \mathrm{~mm}$ Figure 20 . Electron temperature profiles at $Z=170 \mathrm{~mm}$ 


\section{H. Plasma Potential}

Like the plasma density and electron temperature, the plasma potential profiles in the plume also exhibited major changes from the nominal values when the cluster was operated with a single, shared cathode. Figures 21-23 show potentials measured downstream of TH $3 \& 4$ for several different configurations. As shown, operating a single thruster from the 3.2-mm (1/4") Ion Tech cathode located at the center of the cluster caused the peak potential at $\mathrm{Z}=50 \mathrm{~mm}$ to increase to more than 50 volts compared to a normal value of just over 20 volts at this location. Operating both thrusters together with this cathode caused the peak plasma potential to fall to about 35 volts at this location. Similar to the behavior observed in the profiles of number density and electron temperature, coupling two thrusters to a single Busek cathode located in close proximity to one of the devices resulted in plasma potentials nearly identical to the ones recorded with each thruster operating independently. As expected, all of the potentials decreased with increasing axial distance. The relative positions of the curves, however, remained consistent, with the two-thruster, shared central cathode potentials falling between the nominal values and those measured with a single thruster operating from the central cathode.
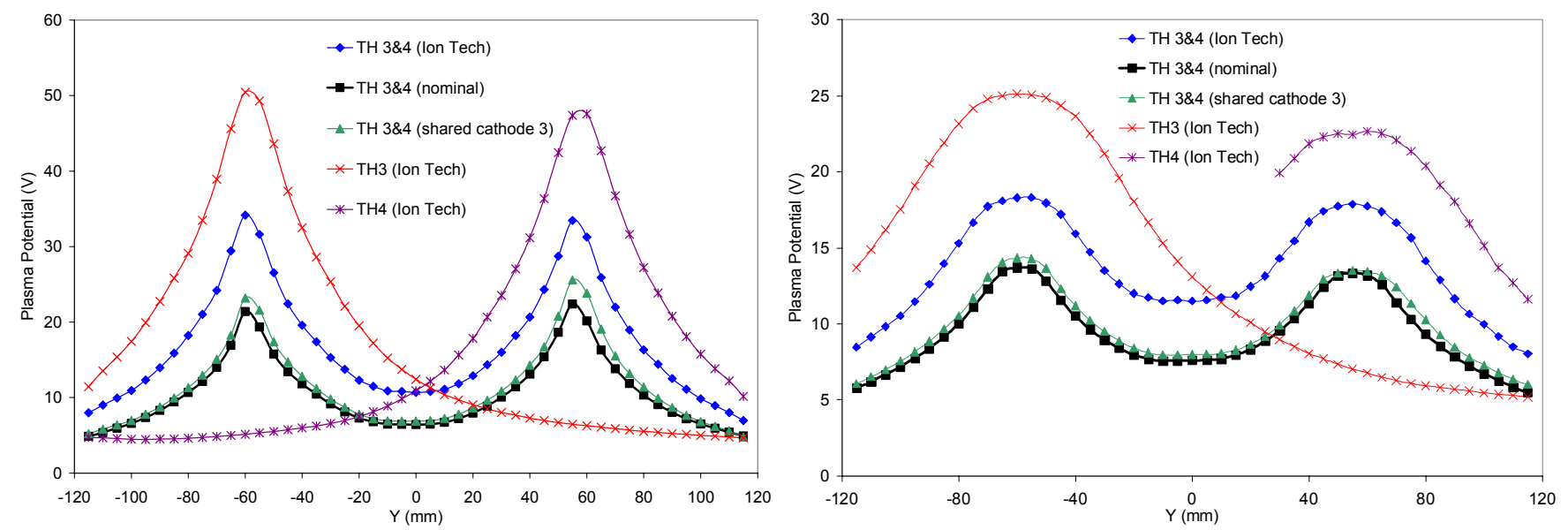

Figure 21. Plasma potential recorded at $Z=50 \mathrm{~mm}$ with $T H$ Figure 22. Plasma potential at $Z=100 \mathrm{~mm}$ measured in $3 \& 4$ coupled to cathode 3 .



Figure 23. Plasma potential at $\mathrm{Z}=150 \mathrm{~mm}$ for various cathode configurations.

As shown in Figs. 24-26, the plasma potential downstream of TH2 was much higher at a given axial location when operated with cathode 3 than it was in the nominal configuration presented previously. ${ }^{6,7}$ Since the boundary conditions of the potential field were set by the applied discharge voltage, these measurements depict a "pushing out" of the plasma potential such that a larger fraction of the potential drop occurred outside of the discharge channel. The stronger electric fields outside of the engine may have a detrimental effect on thruster performance because they can be expected to lead to increased beam divergence. The plots below show that increasing the neutral density, and therefore the particle pressure, 
between the anode and the cathode reduced the potential in the plume somewhat. Doubling the flow through thruster 3 caused a further reduction in the plasma potential, although the difference between the "flow" and "double flow" curves decreased as a function of distance. By about $170 \mathrm{~mm}$ downstream, the two curves became nearly indistinguishable from each other. Finally, compared to the data measured with $8.5 \mathrm{sccm}$ flowing through TH3, energizing electromagnet 3 appeared to cause slight decreases in the plasma potential directly downstream of TH2 and increases in the potential directly downstream of the cathode. The magnitude of the change caused by the magnetic field, however, was relatively small and no definitive conclusions about this effect can be drawn from the collected data (see Section IV). As expected, operating both thrusters together caused the potential in the plume to fall to almost exactly the values measured in the nominal configuration. This behavior is explained in Section IV.

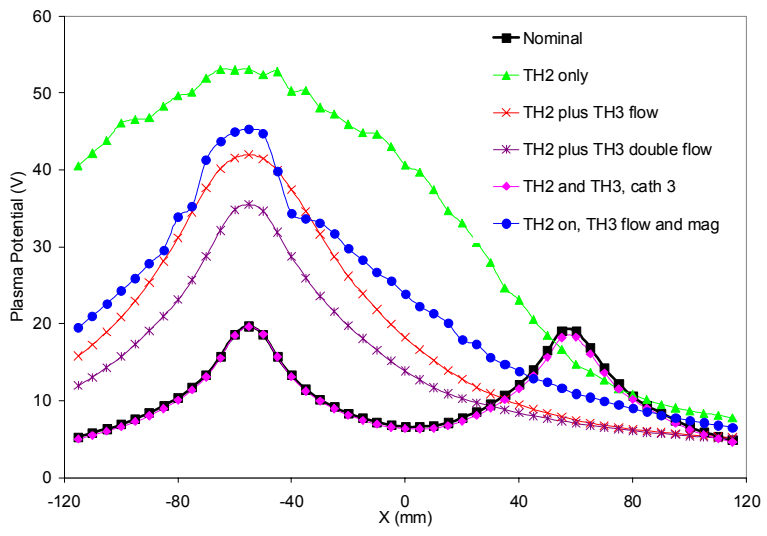

Figure 24. Plasma potential data recorded $70 \mathrm{~mm}$ downstream of TH2 \& TH3. Note the dramatic increase caused by operating a thruster from a distant cathode.

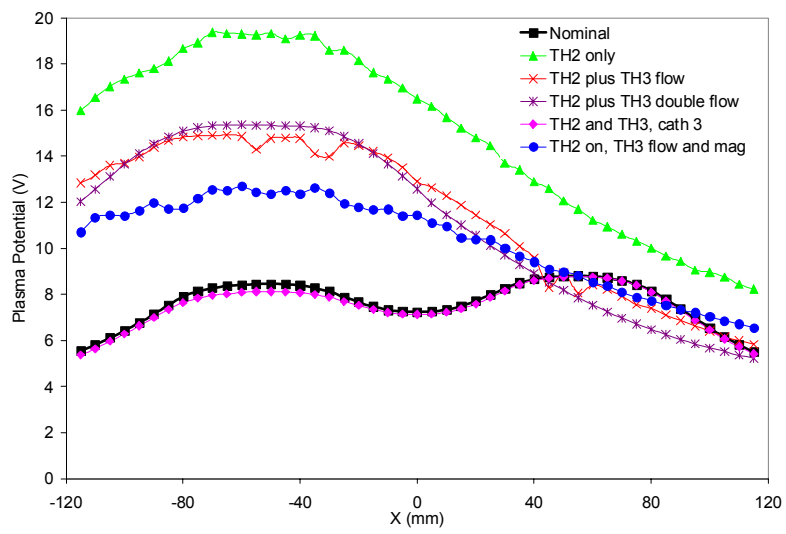

Figure 26. Plasma potential profiles recorded 170 mm downstream of TH2 and TH3 at PEPL.

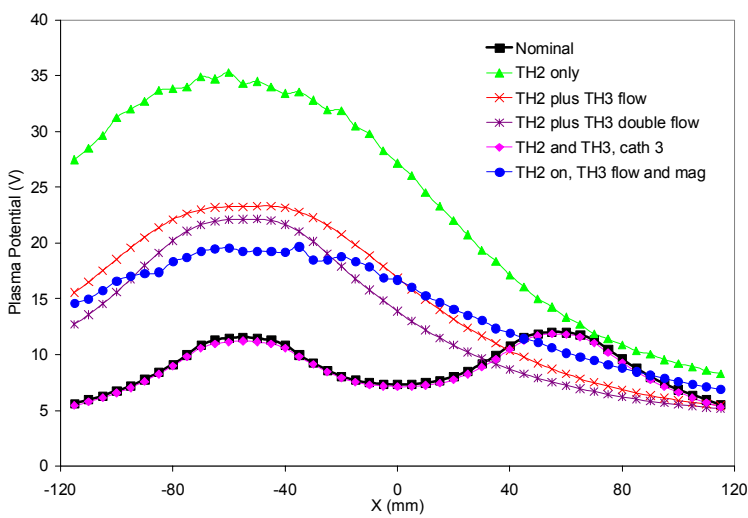

Figure 25. Plasma potential measured $120 \mathrm{~mm}$ downstream of $\mathrm{TH} 2 \&$ TH3 for various cathode configurations. Note the significant reduction in plasma potential caused by adding neutral flow through TH3 (red trace) compared to the case where TH2 is operated with cathode 3 (green trace).

\section{Analysis}

The data presented in the previous sections indicate that the plasma plume properties and basic operating characteristics of a Hall thruster are both influenced by the coupling between the anode and cathode. The most important parameters controlling this process are likely to be the distance between the electrodes and the properties of the medium in the inter-electrode gap. To provide a framework for discussing how these parameters affect the basic operation of the cluster, it is useful to consider the equivalent circuit diagram shown in Fig. 27. This is not meant to imply that the actual transport through the plasma plume is as simple as a purely resistive circuit, but it is 
useful as a qualitative illustration of where the main voltage drops occur in the plume. In this figure, location $\mathrm{P}$ is an arbitrary point in the plume where the plasma potential is measured. The other points are self-explanatory.



Figure 27. A simplified equivalent circuit illustrating the main locations of potential falls in the plume.

Since the discharge voltage, $\mathrm{V}_{\text {dis }}$, was set by the power supply and the cathode potential, $\mathrm{V}_{\text {cath }}$, was observed to be nearly constant (to within a few volts) over the operating conditions of interest, the anode voltage, $\mathrm{V}_{\text {anode }}$, can also be considered constant to a first approximation. Referring to Fig. 27, the plasma potential can be written as Eqn. 5. This shows that the increase in plasma potential observed throughout the plume when a thruster was operated from a distant cathode is indicative of an increase in the effective "resistance" between the cathode and that point in the plume. From the data presented above, it can be concluded empirically that the resistance to electron transport is increased by increasing the distance between the thruster and neutralizer, while it is decreased by increasing the local neutral and plasma densities between the electrodes.

$$
\phi=\frac{R_{\text {far }}}{R_{\text {far }}+R_{\text {near }}+R_{\text {int ernal }}} V_{\text {anode }}
$$

The effect of cathode position and plume properties on the electron transport can be understood by first realizing that the main impediment to electron flow is the magnetic field. Even for the relatively low magnetic field strengths found in the far-field plume (less than 5 gauss), the electron Larmor radius is on the order of several millimeters for temperatures of a few electron volts. To cross the field lines and travel to the anode, the electrons therefore require elastic collisions with other particles. Considering this, it is easy to see that increasing the neutral density or ion density in the cathode region enhances the electron transport by increasing the target population for collisions. Moving the cathode farther away from the thruster, on the other hand, increases the resistance to electron flow by forcing the emitted particles to cross more field lines.

The qualitative description of cathode coupling effects given above can be improved upon by considering the factors influencing electron migration across a magnetic field. Based on the derivation given by Chen, the electron current across the magnetic field can be expressed by Eqn. $6 .{ }^{16}$ In this expression, $v, \mu$, and D represent the total electron collision frequency, electron mobility, and diffusion coefficient, respectively. The subscript $\perp$ is a reminder that it is primarily the quantities perpendicular to the magnetic field that influence the cathode coupling process. As a first approximation, the last term in this expression involving the ExB and diamagnetic drifts can be neglected. It is also helpful to ignore the term involving the density gradient. This will be shown later to have very little effect on our understanding of the processes of interest. With these approximations, Eqn. 6 can be simplified and written as Eqn. 7. 


$$
\begin{aligned}
& \overrightarrow{j_{e \perp}}=-n_{e} e v_{e \perp}=e\left[-n_{e} \mu_{\perp} \vec{E}-D_{\perp} \nabla n_{e}+n_{e} \frac{\overrightarrow{V_{E x B}}+\overrightarrow{V_{D}}}{1+\left(v / \omega_{c}\right)^{2}}\right] \\
& \overrightarrow{j_{e \perp}} \approx-n_{e} e \mu_{\perp} \vec{E}
\end{aligned}
$$

Based on plume properties and discharge current measurements presented elsewhere, ${ }^{6}$ it appears that the electron current to the thruster can be considered nearly constant regardless of the large changes in plasma potential, electron temperature, and electron density observed as a result of varying cathode configuration. This can be deduced by first noting that the discharge current measured with thruster 2 coupled to cathode 3 was only slightly higher than the one measured in the nominal configuration (see, for example, Fig. 8). Second, referring to previously published ion current density traces, ${ }^{6}$ it can be seen that although the shape of the beam profile was changed during operation from a distant cathode, the total ion current exiting the thruster was comparable to the one measured during nominal operation. Since the total discharge current is the sum of both the ion and electron currents, these observations show that the electron current can be considered constant, at least to the low level of accuracy needed to deduce the expected trends from Eqn. 7.

Considering the necessary electron current to the thruster to be a constant, understanding the cathode coupling then reduces to a study of the factors that affect electron mobility. The electron mobility perpendicular to a magnetic field can be expressed by the classical relation given by Eqn. 8. ${ }^{16}$ Even for relatively low magnetic field strengths, the electron cyclotron frequency is much larger than the collision frequency for the densities of interest in the Hall thruster plume. This allows the expression for electron mobility to be simplified by eliminating the collision term in the denominator and replacing the cyclotron frequency with the definition given by Eqn. 9. Combining this result with Eqn. 7 and approximating the electric field to be characterized by the voltage between the cathode and a point in the plume divided by the distance, L, to that point, leads to Eqn. 10.

$$
\begin{gathered}
\mu_{\perp}=\frac{v e / m_{e}}{v^{2}+\omega_{c}^{2}} \\
\omega_{c} \equiv \frac{e B}{m_{e}} \\
n_{e} \frac{m_{e} v}{B^{2}} \frac{\left(\phi+\left|V_{\text {cath }}\right|\right)}{L} \approx \text { const. }
\end{gathered}
$$

The derivation leading to Eqn. 10, although simplistic, provides a theoretical basis for understanding the behavior observed throughout this chapter as the cluster operating conditions were varied. For example, when TH2 was operated from cathode 3 , L was increased compared to the nominal configuration and the plasma potential, $\phi$, increased in response. When flow was added through TH3, the total electron collision frequency, $v$, was increased in the vicinity of the cathode and the plasma potential in the plume decreased as predicted by Eqn. 10. Although this expression is based on far too many simple approximations to be of much use quantitatively, it does explain the trends in plasma potential that can be expected when the cathode position and plume properties near the cathode are varied. The behavior of the electron temperature can, in turn, be understood by realizing that interaction with the electric field is the main source of energy causing heating of the electrons as they flow toward the anode. It follows trivially that anything causing an elevation of the plasma potential in the plume should have a similar effect on the electron temperature, as seen in Figs. 18-20.

One aspect of the observed data that does not, at first glance, appear to be consistent with the trends expected based on Eqn. 10 is the behavior of the plume properties when the electromagnet of TH3 was turned on. According 
to the arguments presented above, activating TH3's electromagnet while TH2 was running would be expected to cause a drastic increase in the plasma potential throughout the plume. The small magnitude of the change in plasma potential measured by the emissive probe in this configuration is believed to be a result of the location of the cathode with respect to TH $2 \& 3$. Referring to Fig. 2, it can be seen that electrons exiting cathode 3 do not need to cross directly in front of TH3 in order to reach TH2, i.e. electrons can flow nearly sideways to TH2 rather than flowing upward to cross the magnetic field lines directly downstream of TH3. Thus, energizing the electromagnet of TH3 does not necessarily cause a significant increase in the number of magnetic field lines that electrons from cathode 3 must cross en route to TH2. Further, since electrons are free to flow parallel to lines of force, it is conceivable that activating TH3's magnet could cause electrons to follow a slightly different path to TH2 without significantly changing the overall impedance through the plasma. This explanation is consistent with the behavior shown in Figs. 24-26 where activating the magnet caused a modest increase in the plasma potential directly downstream of TH3, which was not operating, and a similar decrease downstream of TH2.

Incidentally, the scaling shown in Eqn. 10 relates back to the justification for omitting the diffusion term from the electron transport equation (Eqn. 6). It has been shown that the diffusion coefficient perpendicular to B scales in a way that is very similar to the scaling of the electron mobility, i.e. it is proportional to the collision frequency and inversely proportional to the square of the perpendicular magnetic field. ${ }^{16}$ Thus, the omission of diffusion due to density gradients in the above analysis does not seriously detract from our qualitative understanding of the factors affecting the cathode coupling process.

The changes in plasma properties observed downstream of TH2 as a function of the flow rate through TH3, and whether or not this thruster was operating, can be further illuminated by considering the collision phenomena in the plume. Since it can easily be shown that collisions with like particles do not contribute to electron transport across a magnetic field, ${ }^{16}$ the elastic collision types that influence the cathode coupling process are electron-ion and electronneutral collisions. The characteristic frequencies of these collisions are given by Eqns. 11 and 12 while estimates of the Coulomb logarithm and electron-neutral cross section are given by Eqns. 13 and $14 .{ }^{17}$

$$
\begin{gathered}
v_{e i}=2.9 \times 10^{-12} n_{e} \ln (\Lambda)\left(\frac{k_{b} T_{e}}{e}\right)^{-3 / 2} \\
v_{e n}=\sigma_{e n} n_{n} \sqrt{\frac{k_{b} T_{e}}{m_{e}}} \\
\ln (\Lambda) \cong 23-\frac{1}{2} \ln \left(\frac{10^{-6} n}{\left(k_{b} T_{e} / e\right)^{3}}\right) \\
\sigma_{e n} \approx 6.6 \times 10^{-19}\left\{\frac{k_{b} T_{e} / 4 e^{-0.1}}{1+\left(k_{b} T_{e} / 4 e\right)^{1.6}}\right\}
\end{gathered}
$$

Looking first at collisions between electrons and neutrals, Eqn. 12 gives an estimated collision frequency of about $6 \times 10^{3} \mathrm{~s}^{-1}$ for $2 \mathrm{eV}$ electrons and a neutral density of $5.2 \times 10^{16} \mathrm{~m}^{-3}$. This density is based on the background population that would cause the measured pressure of $1.1 \times 10^{-6}$ Torr when just one thruster was running in the LVTF. When flow was added through thruster 3 , the local neutral density was artificially increased and can be approximated at the exit plane according to Eqn. 15, which assumes that neutrals exit the device at the thermal speed. For a mass flow rate of $0.84 \mathrm{mg} / \mathrm{s}$ and a neutral temperature of $350 \mathrm{~K}$, the estimated neutral density at the exit plane is about $3.2 \times 10^{19} \mathrm{~m}^{-3}$. Taking the characteristic density to be about half of this value (to account for the rapid decrease caused by radial expansion), the electron-neutral collision frequency predicted by Eqn. 11 becomes 
$1.8 \times 10^{6} \mathrm{~s}^{-1}$. The differences in plume properties between operation of TH2 with cathode 3 and operation with flow through thruster 3 are thus the result of increasing the electron collision frequency by more than two orders of magnitude.

$$
n_{n}=\frac{\dot{m}}{m_{i} A_{\text {exit }} \sqrt{\frac{8 k_{b} T_{n}}{\pi m_{i}}}}
$$

The electron-ion collision frequency is estimated by assuming a characteristic plasma density in the region between cathode 3 and thruster 2 . When both $\mathrm{TH} 2$ and $\mathrm{TH} 3$ are operated together, the previously presented data show $5 \times 10^{17} \mathrm{~m}^{-3}$ to be a reasonable estimate. According to Eqn. 11, this leads to an electron-ion collision frequency of approximately $5 \times 10^{6} \mathrm{~s}^{-1}$. This is a very interesting result because it is only about a factor of 3 higher than the estimate of the electron-neutral collision frequency given above, yet operation of thruster 3 (with cathode 3 shared) caused the plume properties to return to approximately the nominal values while just adding flow through thruster 3 caused much smaller changes. This implies that operating multiple thrusters enhances electron transport from the cathode in more ways than by simply increasing the electron collision rate and electron mobility. The mechanism by which electron transport is increased when multiple thrusters are running is not fully understood, but it may be due to a "virtual cathode" effect where the plume of one thruster acts as an electron source for another. In other words, when one thruster is operated from a distant cathode, all of the electrons reaching the anode must originate at the cathode and travel a relatively long distance to reach the anode. When a second thruster is operated in the area between the first thruster and the cathode, the plume electrons from the intermediate device serve as a second source of electrons for the other thruster. Although Kirchoff's laws dictate that all of the electron current must still flow through the hollow cathode, the electrons themselves do not have to travel nearly as far.

One final observation of note regarding the data presented in Section III is the apparent discrepancy between the results presented here and other published measurements. While the data presented here show very pronounced changes in plasma plume properties when a thruster is operated with a distant cathode, both Walker ${ }^{18}$ and Zakharenkov, et al,. ${ }^{19}$ have found that Hall thrusters could be operated with cathodes placed several thruster diameters away with no apparent effect on performance. There are three obvious possibilities that may be considered to explain this. First, since thrust was not measured as part of the present investigation, one could hypothesize that the definitive changes in plasma potential, electron temperature, and plasma density profiles discussed above occurred without being accompanied by a change in performance. Second, since both Walker ${ }^{18}$ and Zakharenkov, et al. ${ }^{19}$ studied larger thrusters, ${ }^{\S}$ it might be reasonable to suppose that larger thrusters are in some way less sensitive to cathode location than the 200-watt engines studied here. Third, it is possible to hypothesize that there may be a particular design feature (not related to power level) that makes certain thrusters more or less sensitive to cathode position. The cause of the discrepancy between the results presented here and those from studies of larger thrusters is not readily apparent from the available data. It is deemed highly unlikely, however, that the rather dramatic changes in plume properties observed in this work could have occurred without a concurrent decline in performance. A parametric study to ascertain why some thrusters appear to be more sensitive to cathode position than others is therefore suggested as a potentially fruitful avenue for further exploration of the characteristics of Hall thruster clusters operating in a shared-cathode configuration.

\section{Implications}

Having examined several of the factors that influence cathode coupling in the Hall thruster plume, it is natural to ask what implications this process has for design and operation of a cluster. Comparing the measurements presented previously for a cluster operating in a modular configuration to those presented in this paper, it is clear that designing a cluster intended for shared cathode operation presents several complications that are not present in the nominal configuration. ${ }^{6,7}$ First, the basic operational characteristics of each thruster using a shared cathode depend on whether or not adjacent thrusters are running. This means that there are likely to be cases where a cluster will

\footnotetext{
${ }^{\S}$ Walker used the 5-kW P5 thruster, Zakharenkov, et al. used a cluster of three 1.5-kW D-55 anode layer thrusters.
} 
perform well when all of the thrusters are running, but operation of a single thruster may result in poor performance due to an inability to efficiently couple the cathode to the plume. This could result in problems for missions that require variable power propulsion systems since operation of a single thruster, such as for station keeping maneuvers, may not be possible if the only available cathode is too far away. Additionally, a system that requires all of the thrusters to be operational to achieve peak performance of any individual engine is inherently vulnerable to single point failures, or at least disproportionate reductions in performance for failure of certain engines. For example, consider the case discussed throughout this chapter where both thrusters 2 and 3 were coupled to cathode 3. Failure of thruster 2 in this configuration would be expected to result in a $50 \%$ reduction in thrust with little or no effect on system efficiency or specific impulse because thruster 3 would still be capable of operating normally. Failure of thruster 3, on the other hand, would result in drastic reductions in system performance because thruster 2 would be incapable of proper operation without thruster 3 running. Obviously, operating multiple devices from a single cathode presents an especially difficult fault tolerance analysis for mission planners and, perhaps, particularly demanding reliability requirements for thruster manufacturers.

In addition to limitations on operational flexibility and fault tolerance, a cluster using a shared cathode presents difficulties for predicting the basic properties of the plume. As shown clearly by the data presented in Section III, the prediction methods used with success in the nominal (modular) configuration do not work when the basic operational characteristics of each thruster depend on the number of engines operating. ${ }^{6}$ This means that a cluster using a shared cathode would need to be ground tested in every conceivable operating mode before it could be used in flight. This may be practical for low-power clusters, but for systems operating at hundreds of kilowatts there exists a very limited number of vacuum facilities capable of supporting full-power testing. Further, the need to test each operating mode individually with the shared cathode partially negates the advantages in development cost and system scalability that were cited as justification for considering a cluster rather than a single, monolithic thruster. For these reasons, the nominal mode discussed elsewhere is likely to be the preferred cluster configuration except, perhaps, in rare situations where the performance benefits associated with shared cathode operation are sufficiently compelling so as to overshadow the difficulties discussed above. ${ }^{6,7}$

\section{Conclusion}

An extensive array of thruster operating parameters and plasma plume properties have been measured for clusters operating in both a parallel configuration and, in another case, with multiple thrusters coupled to a single cathode. The results show that parallel operation tends to allow one cathode to dominate the discharge by emitting the majority of the required electron current. When multiple thrusters are operated in conjunction with a single cathode, however, plume measurements show pronounced differences in plume properties depending on the number of thrusters in operation. In particular, operating a thruster from a distant cathode rather than a local one has been shown to cause increases in plasma potential and electron temperature, as well as a decrease in plasma density, in the near-field plume. When multiple thrusters were operated with a single cathode, the key plume parameters returned to near normal level.

\section{Acknowledgments}

The authors wish to thank Dr. Mitat Birkan of the Air Force Office of Scientific Research and Dr. Ron Spores, formerly of the Air Force Research Laboratory, for financial support. A portion of this work was performed under the auspices of AFOSR Grant F49620-02-1-0051.

\section{References}

${ }^{1}$ Spores, R. A., Spanjers, G. G., Birkan, M., Lawrence, T.J., "Overview of the USAF Electric Propulsion Program," Proceedings of the 37th AIAA Joint Propulsion Conference, AIAA Paper No. 2001-3225, AIAA, Washington, DC, 2001.

${ }^{2}$ Spanjers, G. G., Birkan, M. and Lawrence, T.J., "The USAF Electric Propulsion Research Program," Proceedings of the 36th AIAA Joint Propulsion Conference, AIAA Paper No. 2000-3146, AIAA, Washington, DC, 2000.

${ }^{3}$ Beal, B. E., Gallimore, A. D., and Hargus, W. A., "Preliminary Plume Characterization of a Low-Power Hall Thruster Cluster," Proceedings of the 38th AIAA Joint Propulsion Conference, AIAA Paper No. 2002-4251, AIAA, Washington, DC, 2002.

${ }^{4}$ Hargus, W. A., and Reed, G., "The Air Force Clustered Hall Thruster Program," Proceedings of the 38th AIAA Joint Propulsion Conference, AIAA Paper No. 2002-3678, AIAA, Washington, DC, 2002.

${ }^{5}$ Beal, B. E., and Gallimore, A. D., "Energy Analysis of a Hall Thruster Cluster," Proceedings of the 2003 International Electric Propulsion Conference, IEPC Paper No. 2003-0035, Electric Rocket Propulsion Society, Santa Fe, NM, 2003. 
${ }^{6}$ Beal, B.E., "Clustering of Hall Effect Thrusters for High-Power Electric Propulsion Applications," Ph.D Dissertation, University of Michigan Department of Aerospace Engineering, University Microfilms International, Ann Arbor, MI, 2004.

${ }^{7}$ Beal, B.E., Gallimore, A.D., Hargus, Jr., W.A., "Plasma Properties in the Plume of a Hall Thruster Cluster," Journal of Propulsion and Power, Vol. 20, No. 6, Nov.-Dec. 2004, pp. 985-991.

${ }^{8}$ Hruby, V., Monheiser, J., Pote, B., Rostler, P., Kolencik, J., and Freeman, C., "Development of Low-Power Hall Thrusters," Proceedings of the 1999 AIAA Plasmadynamics and Lasers Conference, AIAA Paper No. 99-3534, AIAA, Washington, DC, 1999.

${ }^{9}$ Chen, S. and Sekiguchi, T., "Instantaneous Direct-Display System of Plasma Parameters by Means of Triple Probe," Journal of Applied Physics, 36, 2363, 1965.

${ }^{10}$ Schott, L., "Electrical Probes," in Plasma Diagnostics, edited by W. Lochte-Holtgreven, American Institute of Physics, Woodbury, NY, 1995.

${ }^{11}$ Hutchinson, I.H., Principles of Plasma Diagnostics, Cambridge University Press, Cambridge, UK, 2002.

${ }^{12}$ Tilley, D. L., Gallimore, A. D., Kelly, A. J., and Jahn, R. G., "The Adverse Effect of Ion Drift Velocity Perpendicular to a Cylindrical Triple Probe," Review of Scientific Instruments (AIP), Vol. 65, No. 3 March 1994, 678-681.

${ }^{13}$ Haas, J.M. and Gallimore, A.D., "Internal Plasma Potential Profiles in a Laboratory Model Hall Thruster," Physics of Plasmas, Vol. 8, No.2, 2001, pp. 652-660.

${ }^{14}$ Kemp, R.F. and Sellen Jr., J.M., "Plasma Potential Measurements by Electron Emissive Probe," Review of Scientific Instruments, Vol. 37, No. 4, 1966, pp. 455-461.

${ }^{15}$ Tilley, D.A., de Grys, K.H., and Myers, R.M., "Hall Thruster - Cathode Coupling," AIAA-99-2865, 35 AIAA/ASME/SAE/ASEE Joint Propulsion Conference \& Exhibit, Los Angeles, CA, June 1999.

${ }^{16}$ Chen, F.F., Introduction to Plasma Physics and Controlled Fusion Volume 1: Plasma Physics, Plenum Press, New York, NY, 1984.

${ }^{17}$ Katz, I., Anderson, J.R., Polk, J.E., and Brophy, J.R., “One-Dimensional Hollow Cathode Model,” Journal of Propulsion and Power, Vol. 19, No. 4, 2003, pp. 595-600.

${ }^{18}$ Walker, M.L.R., "Effects of Facility Backpressure on the Performance and Plume of a Hall Thruster," Ph.D Dissertation, University of Michigan, Department of Aerospace Engineering, University Microfilms International, Ann Arbor, MI, 2005.

${ }^{19}$ Zakharenkov, L.E., Semenkin, A.V., Rusakov, A.V., Urchenko, N.A., Tverdokhlebov, S.O., Garkusha, V.I., Lebedev, U.V., Podkolsin, S.N., Fife, J.M., "Study of Multi Thruster Assembly Operation," Proceedings of the 2003 International Electric Propulsion Conference, IEPC Paper No. 2003-0311, Electric Rocket Propulsion Society, Santa Fe, NM, 2003. 